\title{
Association of cannabis use with prodromal symptoms of psychosis in adolescence
}

Jouko Miettunen, Sari Törmänen, Graham K. Murray, Peter B. Jones, Pirjo Mäki, Hanna Ebeling,

Irma Moilanen, Anja Taanila, Markus Heinimaa, Matti Joukamaa and Juha Veijola

\section{Summary}

Recent interest has focused on the association between cannabis use and risk of psychosis. In the largest unselected, population-based study on this topic to date, we examined cannabis use and prodromal symptoms of psychosis at age 15-16 years among 6330 adolescents. Those who had tried cannabis ( $n=352 ; 5.6 \%$ of the total sample) were more likely to present three or more prodromal symptoms even after controlling for confounders including previous behavioural symptoms (OR=2.23; 95\% Cl 1.70-2.94). A dose-response effect was seen. We conclude that cannabis use is associated with prodromal symptoms of psychosis in adolescence.

\section{Declaration of interest}

None.
Heavy cannabis use is strongly suspected to be associated with both psychotic symptoms and schizophrenia. ${ }^{1}$ Whether cannabis causes psychoses beyond intoxication, however, remains controversial, as some studies describing associations between cannabis use and psychosocial harm are subject to bias or confounding. ${ }^{2}$ Our aim was to examine associations between cannabis use and prodromal symptoms of psychosis in a prospective general population-based birth cohort study.

\section{Method}

The sample was composed of a prospective mother-child birth cohort collected from the two northernmost provinces in Finland. ${ }^{3}$ There were 9340 children ( 4806 boys) alive at the beginning of the latest field study conducted during 2001-2002, when the birth cohort was 15-16 years old. The adolescents were invited to a clinical examination, at which they were given self-completion questionnaires on prodromal symptoms of psychosis and on drug use. Only those who gave informed consent and answered questions on cannabis use were included in the analyses $(n=6298$; 3043 boys). The study was approved by the ethical committee of Oulu University Hospital. Boys (64\% v. 72\% girls), those from urban areas $(64 \% v .72 \%)$ and those from non-intact families $(60 \%$ v. $70 \%)$ were less likely to participate $(P<0.05)$.

For screening for prodromal symptoms of psychosis, we used the PROD-screen, a recently developed questionnaire. ${ }^{4}$ The scale has 21 items; however, we used only the 12 symptom items specifically probing for psychotic-like experiences. ${ }^{5}$ These rate feelings that something strange or inexplicable is taking place in oneself or in the environment, feelings that one is being followed or influenced in some special way, and experience of thoughts running wild or difficulty in controlling the speed of thoughts, among other symptoms. We recorded whether symptoms had been experienced ('no/yes') in the past 6 months. A recommended screening cut-off point of three specific PRODscreen symptoms was used to define cases potentially at risk of developing a psychotic disorder. ${ }^{4}$

Information on early emotional and behavioural symptoms was collected using the Rutter B2 questionnaire for teachers, which measures children's behaviour during the past year. ${ }^{6}$ The scale was completed when the participants were 8 years old. The continuous score from this scale was used as a covariate. Other covariates included gender, family type (both parents all the time $v$. others), parental social class based on occupation (the highest of mother's or father's social class; professionals, entrepreneurs and other white-collar workers $v$. others), regular tobacco use history ('no/yes'), use of other drugs ('no/yes') and parental substance misuse disorder ('no/yes'; information obtained from the nationwide hospital discharge register 1972-2000). We present results using both categorised and continuous symptom variables for a versatile presentation of the data.

\section{Results}

Of the participating adolescents, 352 (5.6\% of the sample) reported that they had ever used cannabis once or more; 59 $(0.9 \%)$ had used cannabis more than five times. Girls reported cannabis use more commonly than boys $(6.1 \%$ v. $4.9 \%$, $P=0.04)$. Those who had tried cannabis had a higher mean number of prodromal symptoms (3.11 v. 1.88 ; $t$-test $=8.68$, $P<0.001)$. When we adjusted for covariates, the difference remained statistically significant. When we analysed categorised prodromal symptoms, those who had tried cannabis were more likely than other adolescents to report three or more prodromal symptoms (crude OR=2.79; 95\% CI 2.24-3.46). For adjusted tests, see Table 1. The proportion of those with high scores increased linearly (OR for linear trend 1.42, 95\% CI 1.23-1.64) by cannabis use category.

\section{Discussion}

Lifetime cannabis use was associated with the incidence of prodromal symptoms of psychosis. This association remained after controlling for confounding factors such as behavioural problems in childhood and other drug use. There was also a dose-response relationship between frequency of cannabis use and increased prodromal symptoms of psychosis. There are some studies supporting this finding of dose-response. ${ }^{2}$

The issue of the prevalence of prodromal symptoms of psychosis among people who use cannabis has not previously been extensively investigated. Schiffman et al found increased schizotypy among undergraduate college students who used cannabis. ${ }^{7}$ They also reported that schizotypal symptoms generally preceded 
Table 1 Prodromal symptoms of psychosis categorised by cannabis use in the sample

\begin{tabular}{|c|c|c|c|}
\hline \multirow[b]{2}{*}{ Cannabis use $^{\mathrm{a}}$} & \multicolumn{2}{|c|}{$\begin{array}{l}\text { PROD-screen } \\
\text { sum score }^{b}\end{array}$} & \multirow{2}{*}{$\begin{array}{c}\text { PROD-screen, } \\
\geqslant 3 \text { symptoms } \\
n(\%)\end{array}$} \\
\hline & $n$ & Mean (s.d.) & \\
\hline Never & 5948 & 1.88 (1.94) & 1749 (29.4) \\
\hline Once & 180 & $2.97(2.53)$ & $94(52.2)$ \\
\hline 2-4 times & 111 & $3.08(2.61)$ & $60(54.1)$ \\
\hline 5 times or more & 50 & $3.68(2.90)$ & $29(58.0)$ \\
\hline Regular use & 9 & $3.11(2.15)$ & $5(55.6)$ \\
\hline \multicolumn{4}{|c|}{$\begin{array}{l}\text { a. Participants were asked the question 'Have you ever tried or used marihuana or } \\
\text { hashish? } \\
\text { b. Test of analysis of covariance (never } v \text {. ever): } F=38.73(P<0.001) \text {. } \\
\text { C. Logistic regression analysis (never } v \text {. ever): OR=2.23 ( } 95 \% \mathrm{Cl} 1.70-2.94) \text {. Tests are } \\
\text { adjusted for the following covariates: early emotional/behavioural symptoms (Rutter } \\
\text { B2 total score), gender, family type, social class, regular tobacco use history, use of } \\
\text { other drugs and parental substance use disorder. }\end{array}$} \\
\hline
\end{tabular}

the onset of cannabis use. ${ }^{7}$ In our study, the association between cannabis use and prodromal symptoms remained even after controlling for early behavioural symptoms at 8 years old.

The PROD-screen use of the term 'prodromal symptoms', the symptom concept and relevant symptom descriptions employed in this paper are in agreement with current international research of clinical markers of psychosis risk. ${ }^{5}$ We note that positive responses on the PROD-screen are not diagnostic of the prodrome of first-episode psychosis since this is a concept that can be established only retrospectively. Nevertheless, the symptoms we measured are consistent with and similar to the prodromal symptoms of established psychosis. There is evidence that psychotic symptoms are much more common in the general population than the incidence of cases of diagnosed psychotic disorder. This can be explained by the continuum hypothesis, according to which psychotic symptoms are expressed on a continuum from mild, clinically irrelevant forms to manifestly psychotic symptoms. ${ }^{8}$ This phenomenon was present in our general population-based study: a large number of the adolescents reported three or more prodromal symptoms. Studying the aetiology of these 'psychotic-like experiences' in the community can illuminate factors contributing to the aetiology of psychotic illness. ${ }^{5}$

Apart from the Swedish conscript study (which included only men), we believe this is the first study in which the association between cannabis use and prodromal symptoms of psychosis has been studied in a large, general population-based sample of adolescents. The participants were adolescents aged 15-16 years, which is a good age at which to investigate the association between cannabis use and psychotic symptoms, as there is evidence that cannabis use at that age is a stronger risk factor for schizophreniform psychosis than later exposure to this substance. ${ }^{9}$ We were able to adjust for several potential confounders including the use of other drugs and early emotional and behavioural problems, and the associations remained significant. The childhood problems were measured at age 8 years by teachers and do not adjust for all subsequent psychopathology that may still pre-date cannabis use. However, teachers' reports have been shown to have good predictive value in earlier studies. ${ }^{10}$ Our sample is more likely to be representative of the general population than many previous studies.
Cannabis use was associated with prodromal symptoms of psychosis in this large Finnish sample of adolescents. We showed these effects are not secondary to confounding effects of other drug use, childhood emotional/behavioural problems or family background. The association is therefore unlikely to be caused by these or any closely related factors, supporting the hypothesis that cannabis use may be causal in terms of subsequent psychotic symptoms. In future studies, we aim to follow the sample and study causal associations between cannabis consumption and psychiatric disorders such as schizophrenia.

Jouko Miettunen, PhD, Department of Psychiatry, University of Oulu and Oulu University Hospital, Oulu, and Academy of Finland, Helsinki; Sari Törmänen, BMed, Department of Psychiatry, University of Oulu and Oulu University Hospital, Oulu, Finland; Graham K. Murray, MRCPsych, Peter B. Jones, FRCPsych, Department of Psychiatry, University of Cambridge, Cambridge, UK; Pirjo Mäki, MD, PhD, Department of Psychiatry, University of Oulu and Oulu University Hospital; Hanna Ebeling, MD, PhD, Irma Moilanen, MD, PhD, Clinic of Child Psychiatry, University of Oulu and Oulu University Hospital; Anja Taanila, PhD, Institute of Health Sciences, University of Oulu, and Unit of General Practice, Oulu University Hospital, Oulu; Markus Heinimaa, MD, Department of Psychiatry, University of Turku, Turku; Matt Joukamaa, MD, PhD, Social Psychiatry Unit, Tampere School of Public Health, Joukamaa, MD, PhD, Social Psychiatry Unit, Tampere School of Public Health,
University of Tampere, and Department of Psychiatry, Tampere University Hospital, Tampere; Juha Veijola, MD, PhD, Department of Psychiatry, University of Oulu and Oulu University Hospital, Oulu, and Academy of Finland, Helsinki, Finland

Correspondence: Dr Jouko Miettunen, Department of Psychiatry, University of Oulu, PO Box 5000, 90014 University of Oulu, Finland. Email: jouko.miettunen@oulu.fi

First received 26 Sep 2007, final revision 18 Jan 2008, accepted 20 Feb 2008

\section{Acknowledgements}

The study was funded by the Academy of Finland, the Signe and Ane Gyllenberg Foundation, the Sigrid Juselius Foundation and the Thule Institute, University of Oulu, Finland.

\section{References}

1 Murray RM, Morrison PD, Henquet C, Di Forti M. Cannabis, the mind and society: the hash realities. Nat Rev Neurosci 2007; 8: 885-95.

2 Moore THM, Zammit S, Lingford-Hughes A, Barnes TRE, Jones PB, Burke M, Lewis G. Cannabis use and risk of psychosis or affective mental health outcomes: a systematic review. Lancet 2007; 370: 319-28.

3 Järvelin MR, Hartikainen-Sorri AL, Rantakallio P. Labour induction policy in hospitals of different levels of specialisation. Br J Obstet Gynaecol 1993; 100: 310-5.

4 Heinimaa $\mathrm{M}$, Salokangas RKR, Ristkari $\mathrm{T}$, Plathin $\mathrm{M}$, Huttunen J, llonen $\mathrm{T}$, Suomela T, Korkeila J, McGlashan TH. PROD-screen - a screen for prodromal symptoms of psychosis. Int J Methods Psychiatr Res 2003; 12: 92-104.

5 Yung AR, Stanford C, Cosgrave E, Killackey E, Phillips E, Nelson B, McGorry PD. Testing the Ultra High Risk (prodromal) criteria for the prediction of psychosis in a clinical sample of young people. Schizophr Res 2006; 84: 57-66.

6 Rutter M. A children's behaviour questionnaire for completion by teachers. J Child Psychol Psychiatr 1967; 8: 1-18.

7 Schiffman J, Nakamura B, Earleywine M, LaBrie J. Symptoms of schizotypy precede cannabis use. Psychiatry Res 2005; 134: 37-42.

8 Verdoux $\mathrm{H}$, van Os J. Psychotic symptoms in non-clinical populations and the continuum of psychosis. Schizophr Res 2002; 54: 59-65.

9 Arsenault L, Cannon M, Poulton R, Murray R, Caspi A, Moffitt TE. Cannabis use in adolescence and risk for adult psychosis: longitudinal prospective study. BMJ 2002; 325: 1212-3.

10 Olin SC, Mednick SA, Cannon T, Jacobsen B, Parnas J, Schulsinger F, Schulsinger $\mathrm{H}$. School teacher ratings predictive of psychiatric outcome 25 years later. Br J Psychiatry 1998; 172 (suppl): 7-13. 